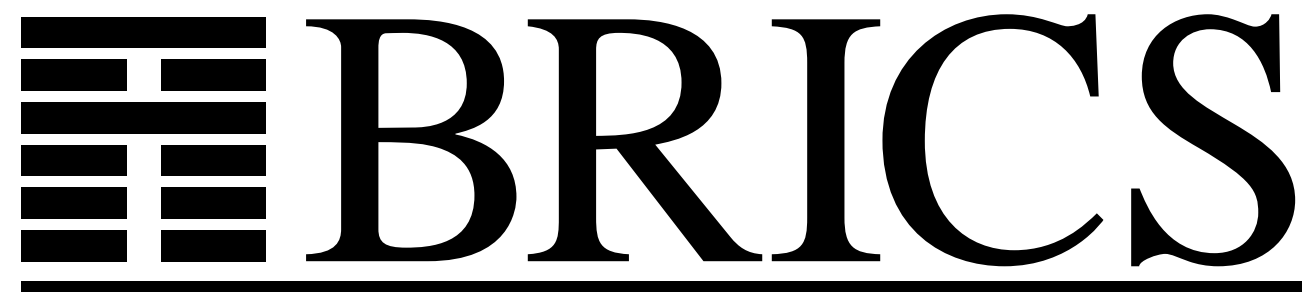

Basic Research in Computer Science

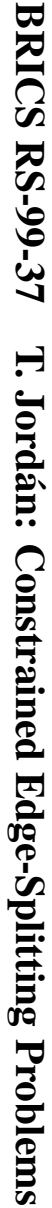

\title{
Constrained Edge-Splitting Problems
}

Tibor Jordán

BRICS Report Series

RS-99-37 
Copyright C 1999, $\quad$ Tibor Jordán.

BRICS, Department of Computer Science University of Aarhus. All rights reserved.

Reproduction of all or part of this work is permitted for educational or research use on condition that this copyright notice is included in any copy.

See back inner page for a list of recent BRICS Report Series publications. Copies may be obtained by contacting:

\author{
BRICS \\ Department of Computer Science \\ University of Aarhus \\ Ny Munkegade, building 540 \\ DK-8000 Aarhus C \\ Denmark \\ Telephone: +4589423360 \\ Telefax: $\quad+4589423255$ \\ Internet: BRICS@brics.dk
}

BRICS publications are in general accessible through the World Wide Web and anonymous FTP through these URLs:

http://www.brics.dk

ftp: / / ftp.brics.dk

This document in subdirectory RS/99/37/ 


\title{
Constrained edge-splitting problems*
}

\author{
Tibor Jordán ${ }^{\dagger}$
}

November 19, 1999

\begin{abstract}
Splitting off two edges $s u, s v$ in a graph $G$ means deleting $s u, s v$ and adding a new edge $u v$. Let $G=(V+s, E)$ be $k$-edge-connected in $V$ $(k \geq 2)$ and let $d(s)$ be even. Lovász proved that the edges incident to $s$ can be split off in pairs in a such a way that the resulting graph on vertex set $V$ is $k$-edge-connected. In this paper we investigate the existence of such complete splitting sequences when the set of split edges has to meet additional requirements. We prove structural properties of the set of those pairs $u, v$ of neighbours of $s$ for which splitting off $s u, s v$ destroys $k$-edgeconnectivity. This leads to a new method for solving problems of this type.

By applying this method we obtain a short proof for a recent result of Nagamochi and Eades on planarity-preserving complete splitting sequences and prove the following new results: let $G$ and $H$ be two graphs on the same set $V+s$ of vertices and suppose that their sets of edges incident to $s$ coincide. Let $G(H)$ be $k$-edge-connected (l-edge-connected, respectively) in $V$ and let $d(s)$ be even. Then there exists a pair $s u, s v$ which can be split off in both graphs preserving $k$-edge-connectivity ( $l$-edge-connectivity, resp.) in $V$, provided $d(s) \geq 6$. If $k$ and $l$ are both even then such a pair always exists. Using these edge-splitting results and the polymatroid intersection theorem we give a polynomial algorithm for the problem of simultaneously augmenting the edge-connectivity of two graphs by adding a (common) set of new edges of (almost) minimum size.
\end{abstract}

*A preliminary version of this paper appeared in: Proc. IPCO '99 (G. Cornuéjols, R.E. Burkard and G. Woeginger, eds.) Springer Lecture Notes in Computer Science 1610, 273-288.

${ }^{\dagger}$ BRICS (Basic Research in Computer Science, Centre of the Danish National Research Foundation), Department of Computer Science, University of Aarhus, Ny Munkegade, building 540, DK-8000 Aarhus, Denmark. e-mail: jordan@daimi .au.dk 
Key words: Edge-splitting, edge-connectivity, graph augmentation, polymatroids, graph algorithms.

AMS subject classification: 05C40, 90C27.

\section{Introduction}

Edge-splitting is a well-known and useful method for solving problems in graph connectivity. Splitting off two edges $s u, s v$ means deleting $s u, s v$ and adding a new edge $u v$. This operation may decrease the edge-connectivity of the graph. The essence of the edge-splitting method is to find a pair of edges which can be split off maintaining the edge-connectivity or other connectivity properties of the graph. If such a good pair exists then one may reduce the problem to a smaller graph which can lead to inductive proofs. Another typical application is the edgeconnectivity augmentation problem where splitting off is an important subroutine in some polynomial algorithms. This connection will be discussed in detail in Section 5. (For a survey see [8].)

Let $G=(V+s, E)$ be a graph which is $k$-edge-connected in $V$, that is, $d(X) \geq k$ holds for every $\emptyset \neq X \subset V$. Here $d(X)$ denotes the degree of $X$. Suppose that $d(s)$ is even and $k \geq 2$. Lovász [11] proved that for every edge $s u$ there exists an edge $s v$ for which splitting off the pair $s u, s v$ maintains $k$-edgeconnectivity in $V$. We call such a pair admissible. By repeated applications of this theorem we can see that all the edges incident to $s$ can be split off in pairs in such a way that the resulting graph (on vertex set $V$ ) is $k$-edge-connected. Such a splitting sequence which isolates $s$ (and preserves $k$-edge-connectivity in $V$ ) is called a complete (admissible) splitting at s.

This result gives no information about the structure of the subgraph $(V, F)$ induced by the set $F$ of new edges that we obtain by the splittings (except the degree-sequence of its vertices, which is the same for every complete splitting). Recent problems in edge-connectivity augmentation gave rise to edge-splitting problems where the goal is to find a complete admissible splitting for which the subgraph of the new edges satisfies some additional requirement. For example, while adding $F$ to $G-s$, one may want to preserve simplicity [2], planarity [12], or bipartiteness [1], too.

The goal of this paper is to develop a new method for solving these kind of edge-splitting problems. The basic idea is to define the non-admissibility graph 
$B(s)$ of $G=(V+s, E)$ on the set of neighbours of $s$ by connecting two vertices $x, y$ if and only if the pair $s x, s y$ is not admissible. We give a complete characterization of those graphs that arise as non-admissibility graphs. Furthermore, we prove that $B(s)$ is 2-edge-connected if and only if $B(s)$ is a cycle, $k$ is odd and $G$ has a special structure which we call round. This structural property turns out to be essential in several edge-splitting problems. Suppose that the additional requirement the split edges have to meet is given by defining a constraint graph $D(s)$ on the neighbours of $s$ at every iteration of the splittings and demanding that for the next admissible pair $s u, s v$ that we split $u v \in E(D(s))$ must hold. Clearly, such an admissible pair exists if and only if $D(s)$ is not a subgraph of $B(s)$. Our method is to compare the structure of the graphs $B(s)$ and $D(s)$ that may occur at some iteration. By showing that $D(s)$ can never be the subgraph of the corresponding $B(s)$ we can verify the existence of a complete admissible splitting satisfying the additional requirement.

As a first application of this method we give simplified proofs for previous results of Nagamochi and Eades [12] on planarity-preserving complete admissible splittings and for some results of Bang-Jensen, Gabow, Jordán and Szigeti [1] on partition-constrained complete admissible splittings. Then we use our structural results to prove the following "intersection theorem" for admissible splittings: let two graphs $G=(V+s, E)$ and $H=(V+s, K)$ be given, for which the sets of edges incident to $s$ in $G$ and $H$ coincide. Let $G$ and $H$ be $k$ - and $l$-edge-connected in $V$, respectively $(k, l \geq 2)$. Then there exists a pair of edges $s u, s v$ which is admissible in $G$ and $H$ (with respect to $k$ and $l$, respectively) simultaneously, provided $d(s) \geq 6$. If $k$ and $l$ are both even then such a pair always exists, and therefore a simultaneously admissible complete splitting exists as well.

Using these edge-splitting results and the $g$-polymatroid intersection theorem of Frank [6] we give a min-max theorem and a polynomial algorithm for the simultaneous edge-connectivity augmentation problem. In this problem two graphs $G^{\prime}=\left(V, E^{\prime}\right), H^{\prime}=\left(V, K^{\prime}\right)$ and two integers $k, l \geq 2$ are given and the goal is to find a smallest set of new edges whose addition makes $G^{\prime}$ (and $H^{\prime}$ ) $k$-edgeconnected ( $l$-edge-connected, respectively) simultaneously. Our algorithm finds a feasible solution whose size does not exceed the optimum by more than one. If $k$ and $l$ are both even then the solution is optimal.

\subsection{Definitions and notation}

Graphs in this paper are undirected and may contain parallel edges. Let $G=$ $(V, E)$ be a graph. A subpartition of $V$ is a collection of pairwise disjoint non- 
empty subsets of $V$. A set consisting of a single vertex $v$ is simply denoted by $v$. An edge joining vertices $x$ and $y$ is denoted by $x y$. Sometimes $x y$ will refer to an arbitrary copy of the parallel edges between $x$ and $y$ but this will not cause any confusion. Adding or deleting an edge $e$ in a graph $G$ is denoted by $G+e$ or $G-e$, respectively. The symbols $\subseteq$ and $\subset$ denote set containment and proper set containment, respectively.

For $X, Y \subseteq V, d(X, Y)$ denotes the number of edges with one endvertex in $X-Y$ and the other in $Y-X$. We define the degree of a subset $X$ as $d(X)=d(X, V-X)$. For example $d(v)$ denotes the degree of vertex $v$. The set of neighbours of $v$ (or v-neighbours, for short), that is, the set of vertices adjacent to vertex $v$, is denoted by $N(v)$. A graph $G=(V, E)$ is $k$-edge-connected if

$$
d(X) \geq k \text { for all } \emptyset \neq X \subset V .
$$

The operation splitting off a pair of edges $s u, s v$ at a vertex $s$ means replacing $s u, s v$ by a new edge $u v$. If $u=v$ then the resulting loop is deleted. We use $G_{u, v}$ to denote the graph obtained after splitting off the edges $s u, s v$ in $G$ (the vertex $s$ will always be clear from the context). A complete splitting at vertex $s$ (with even degree) is a sequence of $d(s) / 2$ splittings of pairs of edges incident to $s$.

\section{Preliminaries}

The degree function satisfies the following well-known equalities.

Proposition 2.1 Let $H=(V, E)$ be a graph. For arbitrary subsets $X, Y \subseteq V$,

$$
\begin{gathered}
d(X)+d(Y)=d(X \cap Y)+d(X \cup Y)+2 d(X, Y), \\
d(X)+d(Y)=d(X-Y)+d(Y-X)+2 d(X \cap Y, V-(X \cup Y)) .
\end{gathered}
$$

In the rest of this section let $s$ be a specified vertex of a graph $G=(V+s, E)$ with degree function $d$ such that $d(s)$ is even and (1) holds with respect to some $k \geq 2$. Saying (1) holds in such a graph $G$ means it holds for all $\emptyset \neq X \subset V$. A set $\emptyset \neq X \subset V$ is called dangerous if $d(X) \leq k+1$ and $d(s, X) \geq 2$. (Notice that in the standard definition of dangerous sets property $d(s, X) \geq 2$ is not required.) A set $\emptyset \neq X \subset V$ is critical if $d(X)=k$. Two sets $X, Y \subseteq V$ are crossing (or $X$ crosses $Y)$ if $X-Y, Y-X, X \cap Y$ and $V-(X \cup Y)$ are all nonempty. Edges $s v, s t$ form an admissible pair in $G$ if $G_{v, t}$ still satisfies (1). It is easy to see that $s v, s t$ is not admissible if and only if some dangerous set contains both $t$ and $v$. 
The following two lemmas can be proved by standard methods using Proposition 2.1. Most of them are well-known and appeared explicitely or implicitely in [7] (or later in [1]). We include the proofs for completeness.

Lemma 2.2 (a) A maximal dangerous set does not cross any critical set.

(b) If $X$ is dangerous then $d(s, V-X) \geq d(s, X)$.

(c) If $k$ is even then two maximal dangerous sets $X, Y$ which are crossing have $d(s, X \cap Y)=0$.

Proof: Let $X$ be a maximal dangerous set and suppose it crosses a critical set $Y$. The maximality of $X$ shows $d(X \cup Y) \geq k+2$. Thus (2) gives $k+1+k \geq$ $d(X)+d(Y) \geq d(X \cap Y)+d(X \cup Y) \geq k+k+2$, a contradiction.

By (1) we see that $k \leq d(V-X)=d(X)-d(s, X)+d(s, V-X)$. Since $d(X) \leq k+1$, this implies $d(s, V-X) \geq d(s, X)-1$ and since $d(s)$ is even we cannot have equality.

Suppose $X$ and $Y$ are maximal dangerous and crossing such that $d(s, X \cap$ $Y) \geq 1$. By Proposition 2.1 and (1), we get that $d(X)=k+1, d(X-Y)=k$ and $d(X \cap Y)=k$. It is easy to see that $d(X)$ is congruent to $d(X-Y)+$ $d(X \cap Y)$ modulo 2, but this contradicts the fact that $d(X)$ is odd and each of $d(X-Y), d(X \cap Y)$ is even.

Let $t$ be a neighbour of $s$. A dangerous set $X$ with $t \in X$ is called a $t$ dangerous set.

Lemma 2.3 Let $v$ be an s-neighbour. Then exactly one of the following holds:

(o) The pair sv, su is admissible for every edge $s u \neq s v$.

(i) There exists a unique maximal $v$-dangerous set $X$.

(ii) There exist precisely two maximal v-dangerous sets $X, Y$. In this case $k$ is odd and we have $d(X)=d(Y)=k+1, d(X-Y)=d(Y-X)=k$, $d(X \cap Y)=k, d(X \cup Y)=k+2, d(X \cap Y, V+s-(X \cup Y))=1, d(s, X-Y) \geq 1$, $d(s, Y-X) \geq 1$, and $d(X \cap Y, X-Y)=d(X \cap Y, Y-X)=(k-1) / 2$.

Proof: Suppose neither (0) nor (i) holds for $v$. Let $\mathcal{F}$ be the collection of maximal $v$-dangerous sets. Any two maximal $v$-dangerous sets are crossing if $d(s)$ is even [7]. Thus $\mathcal{F}$ consists of (at least two) pairwise crossing sets. By Lemma 2.2(c) this shows that $k$ is odd. Let $X, Y \in \mathcal{F}$ be chosen in such a way that $|X \cap Y|$ is as large as possible. Since $X$ and $Y$ cross, by (2) and (3) we obtain $d(X)=d(Y)=k+1, d(X-Y)=d(Y-X)=k, d(X \cap Y)=k$, 
$d(X \cup Y)=k+2, d(X \cap Y, V+s-(X \cup Y))=1$. Thus $d(M)=k$ for $M:=X \cap Y$. Suppose that a set $Z \in \mathcal{F}, Z \neq X, Y$ exists. By Lemma 2.2(a) we can see that $M \subset Z$ and therefore the choice of $X$ and $Y$ implies $M=X \cap Z=Y \cap Z$. From this (3), applied to each pair from $X, Y, Z$ gives $d(M)=1$, contradicting (1). Thus $X$ and $Y$ are the only $v$-dangerous sets. To complete the proof observe that $d(X)=d(X \cap Y)+d(X-Y)-2 d(X \cap Y, X-Y)$ and hence $d(X \cap Y, X-Y)=(k-1) / 2$. Similarly we get $d(X \cap Y, Y-X)=(k-1) / 2$. Our definition of dangerous sets and $d(X \cap Y, V+s-(X \cup Y))=1$ gives $d(s, X-Y) \geq 1$ and $d(s, Y-X) \geq 1$.

An $s$-neighbour $v$ for which (ii) holds in Lemma 2.3 is called special.

The previous lemmas include all ingredients of Frank's proof [7] for the next splitting off theorem due to Lovász.

Theorem 2.4 [11] Suppose that (1) holds in $G=(V+s, E), k \geq 2, d(s)$ is even and $|N(s)| \geq 2$. Then for every edge st there exists an edge su $(t \neq u)$ such that the pair st, su is admissible.

\section{The structure of non-admissibility graphs}

In this section let $G=(V+s, E)$ be given which satisfies (1) with respect to some $k \geq 2$. The non-admissibility graph $B(s)=(N(s), E(B(s)))$ of $G$ (with respect to $s)$ is defined on vertex set $N(s)$. Two vertices $u, v \in N(s),(u \neq v)$ are adjacent in $B(s)$ if and only if the pair $s u, s v$ is not admissible in $G$. Notice that while $G$ may contain multiple edges $B(s)$ is always a simple graph. By definition, two edges $s u, s v(u \neq v)$ form an admissible pair in $G$ if and only if $u v \in E(\bar{B}(s))$, that is, $u v$ is an edge of the complement of $B(s)$.

This notion turns out to be useful in problems where we search for a complete admissible splitting for which the set $F$ of edges obtained by the splittings satisfies some additional property $\Pi$. Let $G^{\prime}:=G-s$. For example, $G^{\prime}+F$ may be required to be simple, planar, or bipartite. If property $\Pi$ is closed under taking subgraphs, it defines a graph for every iteration of a splitting sequence in the following way. Suppose that by splitting off some admissible pairs we have maintained property $\Pi$, that is, $G^{\prime}+F^{\prime}$ satisfies $\Pi$ for the set $F^{\prime}$ of edges split off so far. Define a constraint graph $D_{\Pi}(s)=(N(s), E(D(s)))$ on the set of neighbours of $s$ in such a way that splitting off $x y$ maintains $\Pi$ (that is, $G^{\prime}+F^{\prime}+x y$ satisfies $\Pi$ ) if and only if $x y \in E\left(D_{\Pi}(s)\right)$. By definition, a constraint graph is 
simple. Given $\Pi$ and its constraint graph $D_{\Pi}(s)$, an admissible split satisfying $\Pi$ will be called a $D_{\Pi}(s)$-split, or simply a $D(s)$-split. It is clear from the definitions that a $D(s)$-split exists if and only if $D(s)$ and $\bar{B}(s)$ has a common edge. In other words, a $D(s)$-split does not exist if and only if $D(s)$ is a subgraph of $B(s)$. It is easy to decide whether a $D_{\Pi}(s)$-split exists for a given $\Pi$. On the other hand, to decide whether a complete admissible splitting satisfying property $\Pi$ exists may be difficult (for instance, to decide whether there is a complete admissible splitting in a simple graph that preserves simplicity is NP-complete [10]). Structural properties of the non-admissibility graph help overcome this difficulty in several cases by the following observation: if $D_{\Pi}(s)$ cannot be the subgraph of $B(s)$ at any iteration (since, say, it is always connected while the non-admissibility graph is disconnected) then a complete admissible splitting satisfying property $\Pi$ exists.

In order to use this kind of argument, we characterize those graphs that arise as non-admissibility graphs. A vertex $v$ which is adjacent to all the other vertices of the graph is a dominating vertex. A complete (subgraph of a) graph will be called a clique. The union of two cliques with precisely one vertex in common is a double clique. Every double clique has a dominating vertex. In what follows assume that $d(s)$ is even and $|N(s)| \geq 2$. The definition of $B(s)$, Lemma 2.3 and Theorem 2.4 imply the following.

Lemma 3.1 (a) $B(s)$ has no dominating vertex. (b) The neighbours of a vertex $t$ in $B(s)$ induce a clique, unless $t$ is special. Ift is special, the neighbours oft can be covered by two cliques of $B(s)$.

This leads to a simple characterization of non-admissibility graphs in the case when $k$ is even.

Theorem 3.2 Suppose that $G=(V+s, E)$ satisfies (1), $d(s)$ is even, $|N(s)| \geq 2$, and $k$ is even. Then $B(s)$ is the disjoint union of (at least two) complete graphs.

Proof: By Lemma 2.3 there is no special $s$-neighbour in $G$. Hence, by Lemma 3.1(b), if $s u, s v$ and $s u, s w(v \neq w)$ are both non-admissible then the pair $s v, s w$ is also non-admissible. By this transitivity property $B(s)$ is the union of pairwise disjoint cliques. $B(s)$ itself cannot be complete by Lemma 3.1(a).

It is easy to see that every graph consisting of (at least two) disjoint complete graphs can be obtained as a non-admissibility graph.

Now let us focus on the case when $k \geq 3$ is odd. In this case the complete characterization of non-admissibility graphs is more complicated. We need the following key lemma. 
Lemma 3.3 Suppose that $t$ is special and let $X$ and $Y$ be the two maximal $t$ dangerous sets. Let $u$ be a special s-neighbour in $Y-X$. Let $Y$ and $Z$ denote the two maximal u-dangerous sets in $G$. Then $Z \cap X=\emptyset, Y=(X \cap Y) \cup(Z \cap Y)$ and $d(s, Y)=2$.

Proof: Notice that for every special vertex $u^{\prime} \in Y-X$ one of the two maximal $u^{\prime}$ dangerous sets must be $Y$, thus $Y$ is indeed one of the two maximal $u$-dangerous sets.

Let $v$ be an $s$-neighbour in $Z-Y$. First suppose $v \in X-Y$. Since $X$ and $Y$ are the only maximal $t$-dangerous sets we have $t \notin Z$. This shows that $v$ is also special and the two maximal $v$-dangerous sets are $X$ and $Z$. Lemma 2.3 implies $d(Z)=k+1$ and $d(X-Y)=d(Y-X)=k$. Hence by (3), applied to $Z$ and $X-Y$ and to $Z$ and $Y-X$, we obtain $X-Y \subset Z$ and $Y-X \subset Z$. By Lemma 2.3 we have $d(X \cap Y)=k$. Hence $Z \cap X \cap Y=\emptyset$ by Lemma 2.2, provided $Z \cup(X \cap Y) \neq V$. Moreover, $Z \cup(X \cap Y)=V$ implies $d(s, Z) \geq d(s)-1>d(s, V-Z)$, using $d(s) \geq 4$. Since $Z$ is dangerous, this would contradict Lemma 2.2(b). Thus we conclude $Z \cap X \cap Y=\emptyset$.

The above verified properties of $Z$ and Lemma 2.3 imply that $k+1=d(Z) \geq$ $d(X \cap Y, Z)+d(s, Z) \geq d(X \cap Y, X-Y)+d(X \cap Y, Y-X)+2=k-1+2=k+1$. This shows that $d(s, Z)=2$ and hence $d(s, Z \cup(X \cap Y))=3$ holds. We also get $d(Z, V-Z-(X \cap Y))=0$ and by Lemma 2.3 we have $d(X \cap Y, V-Z-$ $(X \cap Y))=0$ as well. Therefore $d(Z \cup(X \cap Y), V-Z-(X \cap Y))=0$ and hence $d(s, Z \cup(X \cap Y))=d(Z \cup X \cup Y)=3 \leq k$. This shows $Z \cup X \cup Y$ is dangerous, contradicting the maximality of $X$.

Thus we may assume that $v \in V-(X \cup Y)$. By Lemma 2.3 we have $d(Z)=$ $k+1, d(X-Y)=d(Y-X)=d(X \cap Y)=k$ and there exists an $s$-neighbour $w \in X-Y$. Clearly, $t \notin Z$ and by the previous argument we may assume $w \notin Z$. We claim that $Z \cap(X-Y)=\emptyset$. Indeed, otherwise $Z$ and $X-Y$ would cross (observe that $t \notin Z \cup(X-Y)$ ), contradicting Lemma 2.2(a). We claim that $Z \cap(X \cap Y)=\emptyset$ holds as well. This claim follows similarly by Lemma 2.2(a), since $w \notin Z \cup(X \cap Y)$. A third application of Lemma 2.2(a) shows $Y-X \subset Z$. To see this observe that $t \notin Z \cup(Y-X)$ and hence $(Y-X)-Z \neq \emptyset$ would imply that $Z$ and $Y-X$ cross, a contradiction.

Summarizing the previous observations we obtain $Z \cap X=\emptyset$ and $Y=$ $(X \cap Y) \cup(Z \cap Y)$. By Lemma 2.3 this implies $d(s, Y)=2$. This proves the lemma. 
A corollary of Lemma 3.3 is the following sharpening of Lemma 3.1(b). Let $t$ be a special $s$-neighbour and let $X$ and $Y$ be the maximal $t$-dangerous sets. Then each pair $s x, s y$ with $x \in X-Y, y \in Y-X$ is admissible and hence the $t$-neighbours in $B(s)$ induce two disjoint cliques.

We recall some basic definitions and facts. An inclusionwise maximal 2-edgeconnected subgraph is a 2-component. (By definition, a single vertex is 2-edgeconnected.) An edge $e$ is called a cut-edge in a connected graph $H$ if $H-e$ is disconnected. It is well-known that the 2-components of a graph are pairwise vertex-disjoint and those edges of a connected graph which are not included in any 2-component are precisely the cut-edges. By contracting the 2-components of a connected graph $H$ we get a tree whose edges correspond to the cut-edges of $H$. This tree is the 2-component tree of $H$. 2-components corresponding to the leaves of this tree are called leaves.

Theorem 3.4 Suppose that $G=(V+s, E)$ satisfies $(1), d(s)$ is even and $|N(s)| \geq$ 2. Let $H$ be a component of $B(s)$. Then $H$ is either $(a)$ a clique, or $(b)$ a double clique, or $(d)$ two disjoint cliques connected by a path, or $(d)$ a cycle of length at least four.

Proof: First we show that each 2-component $W$ of $H$ is either a clique, or a double-clique, or a cycle. Suppose $W$ is neither a clique nor a double-clique. Since $W$ is not a clique, there exists a vertex $t \in W$ for which the neighbours of $t$ in $W$ do not form a clique. By Lemma 3.1 and the maximality of $W$ it follows that $t$ is special. Let $X$ and $Y$ denote the two cliques in $B(s)$ corresponding to the two maximal $t$-dangerous sets of $G$. By the choice of $t$ and the maximality of $W$ we have $X \cup Y \subseteq W$. Since $X \cup Y$ forms a double-clique in $B(s)$ and $W$ is connected and is not a double-clique, without loss of generality, there is an edge $u v$ in $W$ with $u \in Y-t, v \in W-(X \cup Y)$. Applying Lemma 3.3 to $t$ and $u$ we get $Y=\{t, u\}$ and that $u$ and $t$ have no common neighbours. Let $P$ be a shortest path in $W-t u$ from $t$ to $u$. Such path exists since $W$ is 2-edge-connected. By Lemma 3.3 there are no edges in $B(s)$ from $X-t$ to $Y-t$. Thus $P$ has at least three edges and $C:=P+t u$ is a cycle of length at least four. We claim that $W=C$. Since $P$ is a shortest path, $C$ has no diagonals. Therefore each vertex on $C$ is special by Lemma 3.1(b). Suppose there is a vertex $z \in W-C$ that is adjacent to a vertex of $C$. Since $u$ and $t$ have no common neighbours, we may assume that $z$ and $u$ are nonadjacent. Let $q \neq u$ be the last neighbour of $z$ on $P$ (counting from $t$ to $u$ ) and let $p$ be the vertex preceeding $q$ on $P$. (Observe that $q \neq t$ by Lemma 3.1(b). Thus $p$ exists.) By Lemma 3.1(b) and the choice of $q$, 
it follows that $p$ and $z$ are adjacent. On the other hand, applying Lemma 3.3 to the special vertex $p$ and its neighbour $q$, it follows that $p$ and $q$ have no common neighbours, a contradiction. This proves that $W=C$, as required.

It remains to verify that if $H$ is not 2-edge-connected then it is the union of two disjoint cliques, connected by a path. In our argument the following corollaries of Lemma 3.1(b) and Lemma 3.3, respectively, are crucial: (i) $B(s)$ has no "claw" (that is, a vertex that is adjacent to three pairwise non-adjacent vertices); (ii) $B(s)$ contains no induced subgraph isomorphic to the union of a triangle $(t, w, u)$ and two independent edges $t x, u y$.

Suppose that $H$ is not 2-edge-connected and let $Z$ be a non-singleton 2-component of $H$. By our proof above and since $B(s)$ is simple, $Z$ is either a clique (on at least 3 vertices), or a double-clique (with each clique containing at least 3 vertices), or a cycle (on at least 4 vertices). Since $Z \neq H$, there is at least one cut-edge $e$ incident to $Z$. If $Z$ is a double-clique or a cycle then $Z+e$ has a claw or a bad triangle as in (ii), a contradiction. We have the same conclusion if there are at least two cut-edges incident to $Z$ and $Z$ is a clique. This shows that each non-singleton 2-component of $H$ is a clique which is also a leaf. Therefore, since $B(s)$ has no claw, the 2-component tree must be a path and hence $H$ is the union of two disjoint cliques, connected by a path, as required.

By Lemma 3.1(a) a non-admissibility graph has no dominating vertex. It is not difficult to show that every graph $H^{\prime}$ which has no dominating vertex and for which each component $H$ of $H^{\prime}$ satisfies one of (a)-(d) of Theorem 3.4 arises as the non-admissibility graph $B(s)$ of some graph $G=(V+s, E)$.

Those graphs $G=(V+s, E)$ for which $B(s)$ is 2-edge-connected are of special interest. To describe their structure first we need some definitions. In a cyclic partition $\mathcal{X}=\left(X_{0}, \ldots, X_{t-1}\right)$ of $V$ the $t$ partition classes $\left\{X_{0}, \ldots, X_{t-1}\right\}$ are cyclically ordered. Thus we use the convention $X_{t}=X_{0}$, and so on. In a cyclic partition two classes $X_{i}$ and $X_{j}$ are neighbouring if $|j-i|=1$ and nonneighbouring otherwise. We say that $G^{\prime}=\left(V^{\prime}, E^{\prime}\right)$ is a $C_{l}^{p}$-graph for some $p \geq 3$ and some even $l \geq 2$ if there exists a cyclic partition $\mathcal{Y}=\left(Y_{0}, \ldots, Y_{p-1}\right)$ of $V^{\prime}$ for which $d^{\prime}\left(Y_{i}\right)=l(0 \leq i \leq p-1)$ and $d\left(Y_{i}, Y_{j}\right)=l / 2$ for each pair $Y_{i}, Y_{j}$ of neighbouring classes of $\mathcal{Y}$ (which implies $d\left(Y_{i^{\prime}}, Y_{j^{\prime}}\right)=0$ for each pair of nonneighbouring classes $\left.Y_{i^{\prime}}, Y_{j^{\prime}}\right)$. A cyclic partition of $G^{\prime}$ with these properties is called uniform. 
Let $G=(V+s, E)$ satisfy (1) for some odd $k \geq 3$. Such a $G$ is called round (from vertex $s$ ) if $G-s$ is a $C_{k-1}^{d(s)}$-graph. Note that by (1) this implies that $d\left(s, V_{i}\right)=1$ for each class $V_{i}(0 \leq i \leq d(s)-1)$ of a uniform partition $\mathcal{V}$ of $G-s$.

Lemma 3.5 Let $G=(V+s, E)$ satisfy (1) for some odd $k \geq 3$. Suppose that $G$ is round from $s$ and let $\mathcal{V}=\left(V_{0}, \ldots, V_{r}\right)$ be a uniform partition of $V$, where $r=d(s)-1$. Then

(a) $G-s$ is $(k-1)$-edge-connected and for every $X \subset V$ with $d_{G-s}(X)=$ $k-1$ either $X \subseteq V_{i}$ or $V-X \subseteq V_{i}$ holds for some $0 \leq i \leq r$ or $X=\bigcup_{i}^{i+j} V_{i}$ for some $0 \leq i \leq r, 1 \leq j \leq r-1$.

(b) For any set I of new edges which induces a connected graph on $N(s)$, the graph $(G-s)+I$ is k-edge-connected.

(c) The uniform partition of $G-s$ is unique.

(d) $B(s)$ is a cycle on $d(s)$ vertices (which follows the ordering of $\mathcal{V}$ ).

Proof: Let $H:=G-s$. Since $G$ satisfies $(1)$ and $d_{G}\left(s, V_{i}\right)=1$ for $0 \leq i \leq r$, we get $d_{H}(Y) \geq k-1$ if $Y \subseteq V_{i}$ for some $i$. Suppose that $H$ is not $(k-1)$ edge-connected and let $X \subset V$ be a maximal set with $d_{H}(X) \leq k-2$. By the definition of a uniform partition $X$ cannot be the union of some classes of $\mathcal{V}$. Thus there exists a $V_{j} \in \mathcal{V}$ for which $X$ and $V_{j}$ are intersecting. If $X \cup V_{j}=V$ then $d_{H}(X)=d_{H}(V-X)=d_{H}\left(V_{j}-X\right) \leq k-2$ follows, a contradiction. Thus $X$ and $V_{j}$ cross. Now (2) and the maximality of $X$ imply $k-1+k-2 \geq$ $d_{H}\left(V_{j}\right)+d_{H}(X) \geq d\left(V_{j} \cap X\right)+d\left(V_{j} \cup X\right) \geq k-1+k-1$, a contradiction. This proves $H$ is $(k-1)$-edge-connected. Let $Y \subset V$ satisfy $d_{H}(Y)=k-1$ and assume that neither $Y$ nor $V-Y$ is a subset of some class of $\mathcal{V}$. If $Y$ crosses some $V_{j}$ then by (2) and (3) we have $d_{H}\left(Y \cap V_{j}\right)=d_{H}\left(V_{j}-Y\right)=k-1$. This cannot hold by $(1)$ and $d_{G}\left(s, V_{j}\right)=1$. Thus $Y$ is the union of some classes of $\mathcal{V}$. By the properties of a uniform partition it is clear that these classes have to be consecutive. This proves (a).

Property (a) implies that if $I$ is a set of new edges which induces a connected graph on $N(s)$ then for every $Y \subset V$ with $d_{H}(Y)=k-1$ there is at least one edge $x y \in I$ with $|Y \cap\{x, y\}|=1$. Thus adding $I$ to $H$ increases the edge-connectivity to at least $k$. This proves (b).

To see (c) let us fix some $s$-neighbour $v$. In a given uniform partition this vertex is the unique $s$-neighbour in some $V_{j}$ and by property (a) $V_{j}$ is the unique maximal set of degree $k$ in $G$ which contains $v$. This shows that the set of classes of the uniform partitions is unique. By the degree properties of a uniform partition it follows that the cyclic ordering of these classes is also unique. This proves (c). 
By definition a uniform partition $\mathcal{V}$ satisfies $d_{G}\left(V_{i} \cup V_{i+1}\right)=k+1$ for $0 \leq$ $i \leq r$. Thus $B(s)$ contains a cycle which follows the cyclic ordering of $\mathcal{V}$. This proves (d) when $r=2$. Suppose $r \geq 3$ and take a dangerous set $X$ in $G$. By (a) we can see that $d_{G}(s, X)=2$ and $d_{H}(X)=k-1$ hold and that $X$ must be the union of two consecutive classes of $\mathcal{V}$. Therefore $B(s)$ is a cycle.

Theorem 3.6 Suppose that $G=(V+s, E)$ satisfies (1) for some $k \geq 2$, d(s) is even, and $|N(s)| \geq 2$. Then $B(s)$ is 2-edge-connected if and only if $B(s)$ is a cycle of length $d(s), k$ is odd, $d(s) \geq 4$, and $G$ is round from $s$.

Proof: Suppose that $G$ is round from $s$ and $d(s) \geq 4$. Lemma 3.5(d) shows $B(s)$ is a cycle and hence $B(s)$ is 2-edge-connected. To see the other direction assume that $B(s)$ is 2-edge-connected. By Theorem 3.4 and Lemma 3.1(a) $B(s)$ is a cycle. Thus, by Lemma 3.1(b), each $s$-neighbour is special, and hence there are no parallel edges incident to $s$ by Lemma 2.3. This shows that $B(s)$ has $d(s)$ vertices. Let $v_{0}, \ldots, v_{d(s)-1}$ denote the vertices of $B(s)$, following the cyclic ordering. Let $V_{i}=X_{v_{i}}^{1} \cap X_{v_{i}}^{2}(0 \leq i \leq d(s)-1)$, where $X_{v_{i}}^{1}$ and $X_{v_{i}}^{2}$ are the two maximal $v_{i^{-}}$ dangerous sets in $G$. Now by Lemma 2.3(ii) and Lemma 3.3 it is easy to see that $\left(V_{0}, \ldots, V_{d(s)-1}\right)$ is a uniform partition of $G$. Hence $G$ is round from $s$, as required.

\section{Applications}

In this section we apply Theorems 3.2 and 3.6 and give short proofs for previous results from [1] and [12].

\subsection{Edge-splitting preserving planarity}

The following theorem is due to Nagamochi and Eades [12]. The new proof we present here is substantially simpler.

Theorem 4.1 [12] Let $G=(V+s, E)$ be a planar graph with $d(s)$ even satisfying (1) with respect to either an even $k$ or $k=3$. Then there exists a complete admissible splitting at $s$ for which the resulting graph is planar. 
Proof: Suppose that we are given a fixed planar embedding of $G$. This embedding uniquely determines a cyclic ordering $\mathcal{C}$ of (the edges incident to $s$ and hence) the neighbours of $s$. Clearly, splitting off a pair $s u, s v$ for which $u$ and $v$ are consecutive in this cyclic ordering preserves planarity (and a planar embedding of the resulting graph can be obtained without reembedding $G-\{s u, s v\}$ ). Thus to see that a complete admissible splitting exists at $s$ which preserves planarity it is enough to prove that $(*)$ there exists an admissible pair $s u, s v$ for which $u$ and $v$ are consecutive in $\mathcal{C}$. Let us call such a pair a consecutive admissible pair. By repeated applications of $(*)$ we obtain a complete admissible splitting which preserves planarity (and the embedding of $G-s$ as well).

The existence of a consecutive admissible pair can be formulated in terms of a constraint graph. We may assume $|N(s)| \geq 3$. Let $D(s)$ be a cycle defined on the neighbours of $s$ following the cyclic ordering $\mathcal{C}$. Clearly, a consecutive admissible pair exists if and only if there exists a $D(s)$-split. If $k$ is even then Theorem 3.2 and the fact that $D(s)$ is connected (while the non-admissibility graph $B(s)$ is disconnected) shows that $(*)$ holds in every iteration. Therefore the proof of Theorem 4.1 is complete in the case when $k$ is even. (Note that during the process of iteratively splitting off consecutive admissible pairs the set of neighbours as well as the constraint graph $D(s)$ may change. This happens when $s$ looses some neighbour $w$ by splitting off the last copy of the edges $s w$.)

Now consider the case $k=3$. The above argument and Theorem 3.6 shows (using the fact that $D(s)$ is 2-edge-connected) that by splitting off consecutive admissible pairs as long as possible either we find a complete admissible splitting which preserves planarity (and the embedding of $G-s$ ) or we get stuck in a graph $G^{\prime}$ which is round from $s$ and for which $B_{G^{\prime}}(s)=D_{G^{\prime}}(s)$ holds. In the latter case we need to reembed some parts of $G^{\prime}$ in order to complete the splitting sequence and maintain planarity.

Let us consider such a round $G^{\prime}=\left(V+s, E^{\prime}\right)$ with $B_{G^{\prime}}(s)=D_{G^{\prime}}(s)$. Let $V_{0}, \ldots, V_{2 m-1}$ be the uniform partition of $V$ in $G^{\prime}-s$ (where $2 m:=d_{G^{\prime}}(s)$ ) and let $v_{i}$ be the neighbour of $s$ in $V_{i}(0 \leq i \leq 2 m-1)$. There exists a face $F$ of $G^{\prime}-s$ whose boundary includes every $s$-neighbour. Since $k=3$ and $G^{\prime}$ is round, it can be seen that we may assume that $F$ is a finite face in the embedding of $G^{\prime}-s$ and every edge connecting two consecutive members of the uniform partition of $G^{\prime}-s$ is on the boundary of $F$ as well as on the boundary of the infinite face. Since $G^{\prime}$ is round, Lemma 3.5 applies, and we can easily see that adding the edges $v_{0} v_{m}$ and $v_{i} v_{2 m-i}(1 \leq i \leq m-1)$ to $G^{\prime}-s$ results in a 3 -edge-connected graph $G^{\prime \prime}$. Thus $G^{\prime \prime}$ can be obtained from $G^{\prime}$ by a complete admissible splitting. Furthermore, this set of $m$ new edges can be added to the planar embedding of $G^{\prime}-s$ within face $F$ 
in such a way that in the resulting embedding of $G^{\prime \prime}$ every edge crossing involves the edge $v_{0} v_{m}$. To avoid these edge crossings we can do the following: first we "flip" $V_{0}$ and/or $V_{m}$ in $G^{\prime}-s$, that is, reembed the subgraphs induced by $V_{0}$ and $V_{m}$ in such a way that both $v_{0}$ and $v_{m}$ occur on the boundary of the unbounded face while the edges leaving $V_{0}$ and $V_{m}$ remain unchanged. Since $G^{\prime}$ is round, $k=3$, and $v_{0}$ and $v_{m}$ are $s$-neighbours in $G^{\prime}$, it is easy to see that this can be done. Then we can connect $v_{0}$ and $v_{m}$ within the unbounded face and add the other new edges as before. This yields a planar embedding of $G^{\prime \prime}$. This completes the proof of the theorem.

The theorem does not hold if $k \geq 5$ is odd, see [12]. Note that the above proof implies that the graphs obtained by a maximal planarity preserving admissible splitting sequence are round for every odd $k \geq 5$. The original proof in [12] also used the flipping operation but in a more sophisticated way. Our proof shows that if $k=3$ then at most two flippings are sufficient.

\subsection{Edge-splitting with partition constraints}

Let $G=(V+s, E)$ be a graph for which (1) holds for some $k \geq 2$ and $d(s)$ is even. Let $\mathcal{P}=\left\{P_{1}, P_{2}, \ldots, P_{r}\right\}, 2 \leq r \leq|V|$ be a prescribed partition of $V$. In order to solve a more general partition-constrained augmentation problem, Bang-Jensen, Gabow, Jordán and Szigeti [1] investigated the existence of complete admissible splittings at $s$ for which each split edge connects two distinct classes of $\mathcal{P}$. This problem can also be formulated in terms of constraint graphs and our results on non-admissibility graphs can be applied to obtain somewhat simpler proofs for some (partial) results of [1]. We briefly sketch this connection below. (Note that the partition-constrained edge-splitting problem turns out to be a special case of the "simultaneous edge-splitting problem" that we discuss in detail in Section 5.)

An admissible pair $s x, s y$ is called allowed if $x$ and $y$ belong to different classes of $\mathcal{P}$. Let $S:=N(s), S_{i}:=S \cap P_{i}$ and let $d_{i}:=d\left(s, S_{i}\right)$. The following definition describes a situation when a complete allowed splitting does not exist if $k$ is odd.

Definition 4.2 [1] Let $\left\{A_{1}, A_{2}, B_{1}, B_{2}\right\}$ be a partition of $V$ with the following properties in $G$ for some index $i, 1 \leq i \leq r$ : (i) $d(X)=k$ for $X=A_{1}, A_{2}, B_{1}, B_{2}$; (ii) $d(X, Y)=0$ for $(X, Y)=\left(A_{1}, A_{2}\right),\left(B_{1}, B_{2}\right)$; (iii) $S \cap X=S_{i}$ for $X=$ $A_{1} \cup A_{2}$ or $X=B_{1} \cup B_{2} ;$ (iv) $d_{i}=d(s) / 2$. Such a partition is called a $C_{4^{-}}$ obstacle in $G$. 
The following lemma is extracted from [1, Lemma 2.13, 3.3, 3.4, 5.1].

Lemma 4.3 Let $G=(V+s, E)$ be a graph for which (1) holds, $d(s)$ is even, and $d_{i} \leq d(s) / 2$ for all $1 \leq i \leq r$. Then: (i) if $d(s) \geq 6$ then for any non-empty $S_{i}$ there is an allowed pair sx, sy with $x \in S_{i}$; (ii) if $d(s)=4$ then there exists a complete allowed splitting at $s$ unless $k$ is odd and $G$ contains a $C_{4}$-obstacle $\left\{A_{1}, A_{2}, B_{1}, B_{2}\right\}$. In the latter case in graph $G-s$ we have $d\left(A_{1}\right)=d\left(A_{2}\right)=$ $d\left(B_{1}\right)=d\left(B_{2}\right)=k-1$ and $d\left(A_{1} \cup B_{1}\right)=d\left(A_{1} \cup B_{2}\right)=k-1$. Moreover, (iii) if $k$ is even then there exists a complete allowed splitting at $s$, and (iv) if $k$ is odd then there exists a sequence of allowed splittings of length at least $d(s) / 2-2$.

Proof: For a non-empty $S_{i}$ let the constraint graph $D(s)$ be the complete bipartitite graph on color classes $S_{i}$ and $S-S_{i}$, respectively. Since $d_{i} \leq d(s) / 2$, we have $S-S_{i} \neq \emptyset$. An allowed pair $s x, s y$ with $x \in S_{i}$ exists if and only if there exists a $D(s)$-split. $D(s)$ is either 2-edge-connected and is not a cycle, or has a dominating vertex, or is a four-cycle. In the first two cases Theorem 3.2 and Theorem 3.6 show that a $D(s)$-split exists. Moreover, if $D(s)$ is a four-cycle then $G$ is round and $d(s)=4$. In that case $G-s$ is a $C_{4}$-obstacle with the required properties. This proves (i) and (ii).

Let $d_{j} \geq d_{i}$ for every $1 \leq i \leq r$. Splitting off an allowed pair $s x, s y$ with $x \in P_{j}$ maintains $d_{i} \leq d(s) / 2$ for all $1 \leq i \leq r$. Thus iteratively applying (i) by choosing an $S_{j}$ with the largest $d_{j}$ we can find a complete allowed splitting (if $k$ is even) or a sequence of allowed splittings of length at least $d(s) / 2-2$. This proves (iii) and (iv).

Note that by Lemma 3.5(c) the $C_{4}$-obstacle in (ii), if exists, is unique.

\section{Simultaneous edge-splitting and edge-connectivity augmentation}

In this section we consider the following optimization problem: let $G=(V, E)$ and $H=(V, K)$ be two graphs on the same set $V$ of vertices and let $k, l \geq 2$ be integers. Find a smallest set $F$ of new edges for which $\hat{G}=(V, E+F)$ is $k$-edge-connected and $\hat{H}=(V, K+F)$ is l-edge-connected. Let us call this the simultaneous edge-connectivity augmentation problem. We give a polynomial algorithm which finds an optimal solution if both $k$ and $l$ are even and finds a solution whose size is at most one more than the optimum otherwise. One of the 
two main parts of our algorithm is based on a new splitting off theorem that we prove using Theorems 3.2, 3.4, and 3.6.

If $G=H$ (and $k \geq l$ ) then our problem reduces to finding a smallest set $F$ of edges for which $\hat{G}=(V, E+F)$ is $k$-edge-connected. This is the well-solved $k$-edge-connectivity augmentation problem. Several polynomial algorithms are known which can solve this problem optimally. One approach, which is due to Cai and Sun [3] (simplified and extended later by Frank [7]), divides the problem into two parts: first it extends $G$ by adding a new vertex $s$ and a smallest set $F^{\prime}$ of edges incident to $s$ such that $\left|F^{\prime}\right|$ is even and $G^{\prime}=\left(V+s, E+F^{\prime}\right)$ satisfies (1) with respect to $k$. Then in the second part, using Theorem 2.4, it finds a complete admissible splitting from $s$ in $G^{\prime}$. The resulting set of split edges will be an optimal solution for the $k$-edge-connectivity augmentation problem, see [7].

We follow and extend this approach for the simultaneous augmentation problem. To do this we have to extend both parts: we need an algorithm which finds a smallest $F^{\prime}$ incident to $s$ for which $G^{\prime}=\left(V+s, E+F^{\prime}\right)$ and $H^{\prime}=\left(V+s, K+F^{\prime}\right)$ simultaneously satisfy (1) with respect to $k$ and $l$, respectively, and then we need to verify that there exists a complete splitting at $s$ which is simultaneously admissible in $G^{\prime}$ and $H^{\prime}$.

Both of these extended problems have interesting new properties. While a smallest $F^{\prime}$ in the first part can be found by a greedy deletion procedure in the $k$ edge-connectivity augmentation problem, this is not the case in the simultaneous version. Moreover, a complete splitting at $s$ which is simultaneously admissible in $G^{\prime}$ and $H^{\prime}$ does not always exist. (To see this let $V=\{a, b, c, d\}, E=$ $\{a c, b d\}, K=\{a b, b c, c d, d a\}, F^{\prime}=\{s a, s b, s c, s d\}$ and let $k=2, l=3$.) However, as we shall see, a smallest $F^{\prime}$ can be found in polynomial time by solving an appropriate "submodular flow" problem. Furthermore, if $k$ and $l$ are both even then the required complete and simultaneously admissible splitting does exist (and an "almost complete" splitting sequence can always be found).

\subsection{Simultaneous edge-splitting}

We start with the splitting problem. Let $G=(V+s, E+F)$ and $H=(V+s, K+$ $F$ ) be given which satisfy (1) with respect to $k$ and $l$, respectively. Here $F$ denotes the set of edges incident to $s$. (For simplicity, we assume $V=V(G)=V(H)$, although for the splitting problem we do not need this.) Suppose that $d(s):=$ $d_{G}(s)=d_{H}(s)$ is even. We say that a pair $s u, s v$ is legal if it is admissible in $G$ as well as in $H$. A complete splitting sequence at $s$ is legal if the resulting graphs (after deleting $s$ ) satisfy (1) with respect to $k$ and $l$, respectively. The property of 
being legal can be formulated in terms of a constraint graph $D(s)$. Namely, a pair $s u, s v(u \neq v)$ is legal if and only if $s u, s v$ is a $D(s)$-split in $G$ with respect to $D(s)=\bar{B}_{H}(s)$. Thus a legal pair exists if and only if $\bar{B}_{H}(s)$ is not a subgraph of $B_{G}(s)$.

Lemma 5.1 Let $H=(V+s, K+F)$ satisfy (1) with respect to some $k \geq 2$ and let $d(s)$ be even. Let $D$ be the complement of the non-admissibility graph $B_{H}(s)$ of $H$. Then one of the following holds: (i) $D$ is 2-edge-connected and $D$ is not a cycle, (ii) $D$ has a dominating vertex, (iii) $D=C_{4}$, (iv) $D$ arises from a complete bipartite graph $K_{2, m}(m \geq 1)$ by attaching an edge to a vertex of degree $m$, (v) $D$ consists of two independent edges.

Proof: Let $B=B_{H}(s)$ and let $S:=N(s)$. If $B$ is 2-edge-connected then $B$ is a cycle of length $d(s) \geq 4$ by Theorem 3.6. If $d(s)=4$ then (v) holds, otherwise (i) holds. Thus we may assume that $B$ is not 2 -edge-connected.

Case I: $B$ is disconnected.

If $B$ has an isolated vertex then (ii) holds. Otherwise $S$ has a bipartition $S=$ $X \cup Y,|X|,|Y| \geq 2$, such that there are no edges from $X$ to $Y$ in $B$. Let $p:=|X|$ and $r:=|Y|$. Now $D$ contains a spanning complete bipartite graph $K_{p, r}$. Thus $D$ is 2-edge-connected. If $p=r=2$ then (iii) holds, otherwise (i) holds.

Case II: $B$ is connected (and has at least one cut-edge).

If (at least) one of the components we obtain by deleting an arbitrary cut-edge from $B$ is a sigleton then it is easy to see that $B$ arises from a 2-edge-connected subgraph $M$ by attaching some vertices of degree one. This is impossible by Theorem 3.4 and Lemma 3.1(a). Thus we can assume that there exists an $X \subset S$ with $|X|,|S-X| \geq 2$ and $d_{B}(X)=1$. Let $p:=|X|$ and $r:=|S-X|$ and let $e=x v$, $v \in X$, be the unique edge leaving $X$ in $B$. Now $D$ contains a spanning complete bipartite graph $K_{p, r}$ minus one edge. If $p, r \geq 3$ then (i) holds. If $p=r=2$ then $B$ (as well as $D$ ) is a path on four vertices and hence (iv) holds with $m=1$. Suppose we have $p=2, r \geq 3$. Let $X=\{v, w\}$. Since $B$ is connected, the edge $v w$ is present in $B$. Furthermore, $D-x$ is 2-edge-connected. Thus $d_{D}(x) \geq 2$ implies (i). If $d_{D}(x)=1$ then $x$ is adjacent to every vertex $y \in S-X-\{x\}$ in $B$. Since $v$ has no neighbours in $S-X-\{x\}$, it follows from Lemma 3.1(b) that $x$ is special and $S-X$ induces a complete graph in $B$. Thus $D$ arises from a complete bipartite graph $K_{2, m}$ with $m=r-1 \geq 2$ by attaching an edge (xw) to a vertex of degree $m$. This gives (iv). 
Theorem 5.2 If $d(s) \geq 6$ then there exists a legal pair su, sv. If $k$ and $l$ are both even then there exists a complete legal splitting at $s$.

Proof: Let $D:=\bar{B}_{H}(s)$ and $A:=B_{G}(s)$. If $k$ and $l$ are both even then Theorem 3.2 shows that $D$ is connected (since it is the complement of a disconnected graph) and $A$ is disconnected. This implies that a legal pair exists for arbitrary even $d(s)$ and hence a complete legal splitting exists as well.

Suppose $k$ is odd and $d(s) \geq 6$. We may assume $|N(s)| \geq 4$. (Otherwise, by Lemma 3.1(a), $B_{H}(s)$ has an isolated vertex and hence $D$ has a dominating vertex, while $A$ has no such vertex.) $D$ satisfies one of (i)-(v) in Lemma 5.1. If (i) or (ii) holds then by Theorem 3.6 and Lemma 3.1(a) $D$ cannot be a subgraph of $A$ and hence we are done. If (v) holds then $B_{H}(s)$ is cycle of length four. If (iii) holds and $D$ is a subgraph of $A$ then $A$ is 2-edge-connected and by Theorem 3.6 we have that $A$ is a cycle of length four. In both cases $d(s)=4$ follows, a contradiction.

Now assume (iv) holds. If $m=1$ then $D$ (as well as $B$ ) is a path on four vertices. In this case if $A$ contains $D$ then by Lemma 3.1(a) either $A$ is a fourcycle, in which case $d(s)=4$ follows, or $A=D$. In the latter case Lemma 3.1(b) implies that the two inner vertices of $A$ are special in $G$. Similarly, the two inner vertices of $B$ (which are disjoint from the inner vertices of $A$ ) are special in $H$. Therefore by Lemma 2.3 there are no parallel edges incident to $s$ and $d(s)=4$. This settles case (iv) when $m=1$.

If (iv) holds with $m \geq 2$ then $A$ cannot be a cycle. Thus by Theorem $3.6 A$ is not 2-edge-connected. On the other hand, if $A$ contains (the 2-connected graph) $D$ then by Theorem $3.4 A$ can be obtained from a cycle by attaching an edge. This contradicts Lemma 3.1(b). This completes the proof of the theorem.

Corollary 5.3 Suppose that $d(s)=4$ and there exists no legal pair. Then at least one of $G$ and $H$ is round.

Proof: In the proof of Theorem 5.2 we already saw that if no legal pair exists then $d(s)=4$ and either one of $G$ and $H$ is round or $B_{H}(s)$ is a path on four vertices. We show the latter case is impossible. Let the path be $(a, b, c, d)$. By the definition of $B_{H}(s)$, there exists a dangerous set $X$ in $H$ which contains $b$ and $c$. Since $d(s)=|N(s)|=4$, Lemma 2.2(b) implies $d(s, X)=d(s, V-X)=2$. Therefore $d(X)=d(V-X), V-X$ is also dangerous, and $a, d \in V-X$. In this case $a$ and $d$ should also be adjacent in $B_{H}(s)$, a contradiction. 
Note that a complete splitting sequence which is simultaneously admissible in three (or more) graphs does not necessarily exist, even if each of the edgeconnectivity values is even.

We remark that the partition-constrained splitting problem can be reduced to a simultaneous edge-splitting problem where at least one of $k$ and $l$ is even. To see this suppose that an instance of the partition-constrained splitting problem is given as in the beginning of Section 4.2. Let $d_{m}:=\max _{i}\left\{d_{G}\left(s, P_{i}\right)\right\}$ in $G=$ $(V+s, E+F)$ and let $S:=N_{G}(s)$. Build graph $H=(S+x+s, K+F)$ as follows. For each set $S \cap P_{i}$ in $G$ let the corresponding set in $H$ induce a $\left(2 d_{m}\right)$ edge-connected graph (say, a complete graph with sufficiently many parallel edges or a singleton). The edges incident to $s$ in $G$ and $H$ coincide. Then from vertex $x$ of $H$ add $2 d_{m}-d_{G}\left(s, P_{i}\right)$ parallel edges to some vertex of $S \cap P_{i}(1 \leq i \leq r)$. Now $H$ satisfies (1) with respect to $l:=2 d_{m}$. It can be seen that a complete admissible splitting satisfying the partition-constraints in $G$ exists if and only if there exists a complete legal splitting in the pair $G, H$. This shows that characterizing the pairs $G, H$ for which a complete legal splitting does not exist (even if one of $k$ and $l$ is even) is at least as difficult as the solution of the partition-constrained problem [1].

\subsection{Simultaneous edge-connectivity augmentation}

Let $G=(V, E)$ and $H=(V, K)$ be given for which (1) holds with respect to $k$ and $l$, respectively. First we show how to find a smallest $F^{\prime}$ for which the extended graphs $G^{\prime}=\left(V+s, E+F^{\prime}\right)$ and $H^{\prime}=\left(V+s, K+F^{\prime}\right)$ simultaneously satisfy (1) with respect to $k$ and $l$, respectively. We need some results from the theory of polymatroids.

Let $V$ be a finite ground-set and let $p: 2^{V} \rightarrow Z \cup\{-\infty\}$ be an integer-valued function for which $p(\emptyset)=0$. We call $p$ fully supermodular if $p(X)+p(Y) \leq$ $p(X \cap Y)+p(X \cup Y)$ holds for every $X, Y \subseteq V$. A function $p: 2^{V} \rightarrow Z \cup\{-\infty\}$ is skew supermodular if for every $X, Y \subseteq V$ either the above submodular inequality holds or $p(X)+p(Y) \leq p(X-Y)+p(Y-X)$. If $p(Y) \leq p(X)$ holds for every $Y \subseteq X$ then $p$ is monotone. For a fully supermodular and monotone function $p$ the set $C(p):=\left\{x \in R^{V}: x \geq 0, x(A) \geq p(A)\right.$ for every $\left.A \subseteq V\right\}$ is called the contra-polymatroid of $p$. The next result is due to Frank. 
Theorem 5.4 [7] Let $p$ be a skew supermodular function. Then $C(p)$ is a contrapolymatroid whose unique (monotone, fully supermodular) defining function $\bar{p}$ is given by

$$
\bar{p}(X):=\max \left(\sum_{t} p\left(X_{i}\right):\left\{X_{1}, \ldots, X_{t}\right\} \text { is a subpartition of } X\right) .
$$

Given a graph $G=(V, E)$ and $k \in Z_{+}$, let $p_{G}^{k}: 2^{V} \rightarrow Z$ be defined by $p_{G}^{k}(X):=k-d_{G}(X)$, if $\emptyset \neq X \neq V$ and $p_{G}^{k}(\emptyset)=p_{G}^{k}(V)=0$. This function $p_{G}$ is skew-supermodular by Proposition 2.1. Following [7], we say that a vector $z: V \rightarrow Z_{+}$is an augmentation vector of $G$ (with respect to $k$ ) if $z(X) \geq p_{G}^{k}(X)$ for every $X \subseteq V$. Observe that $G^{\prime}=(V+s, E+F)$ satisfies (1) with respect to $k$ if and only if $z(v):=d_{F}(v)(v \in V)$ is an augmentation vector. Hence by Theorem 5.4 the problem of finding a smallest $F$ for which $G^{\prime}=(V+s, E+F)$ satisfies (1) can be reduced to finding an integer valued element of the contrapolymatroid $C\left(p_{G}^{k}\right)$ for which $z(V)$ is minimum. This can be done by a greedy algorithm [7]. Similarly, adding $F^{\prime}$ is simultaneously good for $G$ and $H$ if and only if $z(X) \geq \max \left\{p_{G}^{k}(X), p_{H}^{l}(X)\right\}$ for every $X \subseteq V$, where $z(v):=d_{F^{\prime}}(v)$, $(v \in V)$. Let us call such a $z$ a common augmentation vector of $G$ and $H$. Clearly, finding a smallest $F^{\prime}$ for which $G^{\prime}=\left(V+s, E+F^{\prime}\right)$ and $H^{\prime}=(V+$ $\left.s, K+F^{\prime}\right)$ satisfy (1) with respect to $k$ and $l$, respectively, can be solved by finding an integer valued $z \in C\left(p_{G}\right) \cap C\left(p_{H}\right)$ for which $z(V)$ is minimum. By Frank's [6] " $g$-polymatroid intersection theorem" (see also Frank and Tardos [9, Theorem 1.4, Proposition 4.1] and [5]) the system $\mathcal{S}\left(p_{G}^{k}, p_{H}^{l}\right)=\left\{x \in R^{V}: x \geq\right.$ $\left.0, x(A) \geq \max \left\{p_{G}^{k}(A), p_{H}^{l}(A)\right\}\right\}$ is a "submodular flow system" and hence we can find an integer valued $x \in \mathcal{S}\left(p_{G}^{k}, p_{H}^{l}\right)$ minimizing $x(V)$ in polynomial time (see e.g. Cunningham and Frank [4]).

Summarizing our observations we obtain the following algorithm for the simultaneous edge-connectivity augmentation problem. Let $G=(V, E)$ and $H=$ $(V, K)$ (satisfying (1) with respect to $k$ and $l$, resp.) be the pair of input graphs.

(Step 1) Find a common augmentation vector for $G$ and $H$ for which $z(V)$ is as small as possible.

(Step 2) Add a new vertex $s$ to each of $G$ and $H$ and $z(v)$ parallel edges from $s$ to $v$ for every $v \in V$. If $z(V)$ is odd then add one more edge $s w$ for some $w \in V$.

(Step 3) Find a maximal legal splitting sequence $\mathcal{S}$ at $s$ in the resulting pair of graphs. If $\mathcal{S}$ is complete, let the solution $F$ consist of the set of split edges. Otherwise splitting off $\mathcal{S}$ results in a pair of graphs $G^{\prime}, H^{\prime}$ for which $d(s)=|N(s)|=4$. In this case let the solution $F$ be the union of the split edges and a set $I$ of three properly chosen additional edges that form a path on the four $s$-neighbours. 
The following theorem shows the correctness of the above algorithm and proves that the solution set $F$ is (almost) optimal. Let us define

$$
\begin{gathered}
\Phi_{k, l}(G, H)=\max \left\{\sum_{1}^{r}\left(k-d_{G}\left(X_{i}\right)\right)+\sum_{r+1}^{t}\left(l-d_{H}\left(X_{i}\right):\right.\right. \\
\left.\left\{X_{1}, \ldots, X_{t}\right\} \text { is a subpartition of } V ; 0 \leq r \leq t\right\} .
\end{gathered}
$$

The size of a smallest simultaneous augmenting set for $G$ and $H$ (with respect to $k$ and $l$, resp.) is denoted by $O P T_{k, l}(G, H)$.

Theorem $5.5\left\lceil\Phi_{k, l}(G, H) / 2\right\rceil \leq O P T_{k, l}(G, H) \leq\left\lceil\Phi_{k, l}(G, H) / 2\right\rceil+1$. If $k$ and $l$ are both even then $O P T_{k, l}(G, H)=\left\lceil\Phi_{k, l}(G, H) / 2\right\rceil$ holds.

Proof: It is easy to see that $\left\lceil\Phi_{k, l}(G, H) / 2\right\rceil \leq O P T_{k, l}(G, H)$ holds. We shall prove that the above algorithm results in a simultaneous augmenting set $F$ with size at most $\left\lceil\Phi_{k, l}(G, H) / 2\right\rceil+1$ (and with size $\left\lceil\Phi_{k, l}(G, H) / 2\right\rceil$, if $k$ and $l$ are both even). ¿From the $g$-polymatroid intersection theorem, Theorem 5.4 and our remarks on common augmentation vectors it follows that for the vector $z$ that we obtain in Step 1 of the above algorithm we have $z(V)=\Phi_{k, l}(G, H)$. Hence we have $2\left\lceil\Phi_{k, l}(G, H) / 2\right\rceil$ edges incident to $s$ at the end of Step 2. By Theorem 5.2 we can find a maximal sequence of legal splittings in Step 3 which is either complete or results in a pair of graphs $G^{\prime}, H^{\prime}$, where $d(s)=|N(s)|=4$. In the former case the set $F$ of split edges, which is clearly a feasible simultaneous augmenting set, has size $\left\lceil\Phi_{k, l}(G, H) / 2\right\rceil$, and hence is optimal. If $k$ and $l$ are both even then such a complete legal splitting always exists, proving $O P T_{k, l}(G, H)=\left\lceil\Phi_{k, l}(G, H) / 2\right\rceil$. In the latter case one of $G^{\prime}$ and $H^{\prime}$, say $G^{\prime}$, is round by Corollary 5.3. There exists a complete admissible splitting in $H^{\prime}$ by Theorem 2.4. Let $e=u v, f=x y$ be the two edges obtained by such a complete splitting. Let $g=v x$. Adding $e$ and $f$ to $H^{\prime}-s$ gives an $l$-edge-connected graph and by Lemma 3.5(b) adding the edge set $I:=\{e, f, g\}$ to $G^{\prime}-s$ yields a $k$-edge-connected graph. Thus the set of edges $F$ which is the union of the edges obtained by the maximal legal splitting sequence and the edge set $I$ is a simultaneous augmenting set. We also have $|F|=\left\lceil\Phi_{k, l}(G, H) / 2\right\rceil+1$, as required.

There are examples showing $O P T=\lceil\Phi / 2\rceil+1$ may hold. (Take $V=$ $\{a, b, c, d\}, E=\{a c, b d\}, K=\{a b, b c, c d, d a\}$, and let $k=2, l=3$.) It is easy to see that the above algorithm can be implemented in polynomial time. As we pointed out, Step 1 is a submodular flow problem and hence can be solved in polynomial time. One approach to solve Step 3 efficiently is using max-flow computations to check whether a pair of edges is legal or not. We omit the algorithmic details. 


\section{Acknowledgements}

I thank Bill Jackson for several useful comments.

\section{References}

[1] J. Bang-Jensen, H.N. Gabow, T. Jordán and Z. Szigeti, Edge-connectivity augmentation with partition constraints, SIAM J. Discrete Mathematics Vol. 12, No. 2, 160-207 (1999).

[2] J. Bang-Jensen, T. Jordán, Edge-connectivity augmentation preserving simplicity, SIAM J. Discrete Mathematics Vol. 11, No. 4, 603-623 (1998).

[3] G.R. Cai and Y.G. Sun, The minimum augmentation of any graph to a $k$ edge-connected graph, Networks 19 (1989), 151-172.

[4] W.H. Cunningham, A. Frank, A primal-dual algorithm for submodular flows, Mathematics of Operations Research, 10 (1985) 251-262.

[5] J. Edmonds, R. Giles, A min-max relation for submodular functions on graphs, Annals of Discrete Mathematics 1 (1977) 185-204.

[6] A. Frank, Generalized polymatroids, in Finite and infinite sets, A. Hajnal et al., eds., North-Holland, Amsterdam, 1984, pp. 285-294.

[7] A. Frank, Augmenting graphs to meet edge-connectivity requirements, SIAM J. Discrete Mathematics, 5 (1992) 22-53.

[8] A. Frank, Connectivity augmentation problems in network design, in: Mathematical Programming: State of the Art 1994, (Eds. J.R. Birge and K.G. Murty), The University of Michigan, Ann Arbor, MI, 34-63, 1994.

[9] A. Frank and É. Tardos, Generalized polymatroids and submodular flows, Mathematical Programming 42, 1988, pp 489-563.

[10] T. Jordán, Two NP-complete augmentation problems, IMADA, Odense Universitet Preprints No. 8/1997. http://www.imada.sdu.dk/Research/Preprints /

[11] L. Lovász, Combinatorial Problems and Exercises, North-Holland, Amsterdam, 1979. 
[12] H. Nagamochi and P. Eades, Edge-splitting and edge-connectivity augmentation in planar graphs, R.E. Bixby et al., eds., IPCO VI, Springer LNCS 1412, pp. 96-111, 1998. (Full paper: Department of Applied Mathematics and Physics, Kyoto University, Tech. Report 97007, 30 pages. http://www.kuamp.kyoto-u.ac.jp/) 


\section{Recent BRICS Report Series Publications}

RS-99-37 Tibor Jordán. Constrained Edge-Splitting Problems. November 1999. 23 pp. A preliminary version with the title Edge-Splitting Problems with Demands appeared in Cornujols, Burkard and Woeginger, editors, Integer Programming and Combinatorial Optimization: 7th International Conference, IPCO '99 Proceedings, LNCS 1610, 1999, pages 273-288.

RS-99-36 Gian Luca Cattani and Glynn Winskel. Presheaf Models for CCS-like Languages. November 1999.

RS-99-35 Tibor Jordán and Zoltán Szigeti. Detachments Preserving Local Edge-Connectivity of Graphs. November 1999. 16 pp.

RS-99-34 Flemming Friche Rodler. Wavelet Based 3D Compression for Very Large Volume Data Supporting Fast Random Access. October 1999. $36 \mathrm{pp}$.

RS-99-33 Luca Aceto, Zoltán Ésik, and Anna Ingólfsdóttir. The MaxPlus Algebra of the Natural Numbers has no Finite Equational Basis. October 1999. 25 pp. To appear in Theoretical Computer Science.

RS-99-32 Luca Aceto and François Laroussinie. Is your Model Checker on Time? - On the Complexity of Model Checking for Timed Modal Logics. October 1999. 11 pp. Appears in Kutyłowski, Pacholski and Wierzbicki, editors, Mathematical Foundations of Computer Science: 24th International Symposium, MFCS '99 Proceedings, LNCS 1672, 1999, pages 125-136.

RS-99-31 Ulrich Kohlenbach. Foundational and Mathematical Uses of Higher Types. September 1999. 34 pp.

RS-99-30 Luca Aceto, Willem Jan Fokkink, and Chris Verhoef. Structural Operational Semantics. September 1999. 128 pp. To appear in Bergstra, Ponse and Smolka, editors, Handbook of Process Algebra, 1999.

RS-99-29 Søren Riis. A Complexity Gap for Tree-Resolution. September 1999. 33 pp.

RS-99-28 Thomas Troels Hildebrandt. A Fully Abstract Presheaf Semantics of SCCS with Finite Delay. September 1999. 37 pp. To appear in Category Theory and Computer Science: 8th International Conference, CTCS '99 Proceedings, ENTCS, 1999. 